\title{
EL ARCO IRIS, ¿ES UN TRATADO DE FÍSICA?
}

\section{THE RAINBOW, IS A TREATISE ON PHYSICS?}

José Antonio Martínez Pons: Departamento de Química Analítica e Ingeniería Química. Universidad de Alcalá de Henares. Madrid (España). joseantonio.martines@uah.es

\section{CURRÍCULUM VITAE}

Licenciado en Ciencias Físicas (Geofísica) por la Universidad Complutense de Madrid (España) y en Ciencias Químicas (Química física) por la UNED (España). Doctor por la Universidad de Alcalá de Henares (España) en el año 2000. Profesor de la Universidad de Alcalá de Henares en el área de Química Analítica e Ingeniería Química.

\section{RESUMEN}

El Arco Iris es un fenómeno de la naturaleza. Pero la explicación de por qué se produce es bastante compleja y no suele encontrarse en los libros de Física elemental. Lo normal es que aparezca sólo un arco, pero hay veces que aparecen dos, uno más intenso que el otro. En este artículo se dan varias explicaciones del porqué del Arco Iris. Una según Aristóteles, otra según los arcos supernumerarios de Young, y la última según Airy, basada en los conocimientos que se tenían sobre las propiedades de las ondas en la época. 


\section{PALABRAS CLAVE}

Arco Iris - Física - Aristóteles - Ondas

\section{ABSTRACT}

The Rainbow is a freak of nature. But the explanation of why it occurs is quite complex and not often found in elementary physics books. Typically, you see only a bow, but sometimes there are two, one more intense than the other. This article gives several explanations of why the Rainbow. A according to Aristotle, another supernumerary arcs according to Young, and the last by Airy, based on the knowledge to be had on the properties of waves at the time.

\section{KEY WORDS}

Rainbow - Physics - Aristotle - Waves

\section{ÍNDICE}

1. Resumen

2. Introducción

3. Primeras observaciones

4. Primer nivel de explicación

5. Segundo nivel de explicación

6. Tercer nivel de explicación

\section{Conclusión}

8. Agradecimientos

9. Notas

\section{TEXTO:}




\section{Resumen}

Con ser un fenómeno de la naturaleza de sobra conocido, el arco iris tiene una explicación compleja a la que se puede llegar por aproximaciones sucesivas y que no suele encontrarse en los libros de Física elemental. En este trabajo, pensado como apoyo del profesor no especialista, se reproducen viejos estudios utilizando tecnología actual y se proponen algunas pautas de trabajo que faciliten la comprensión del fenómeno y el aprovechamiento didáctico del mismo.

"Ved aquí la señal del pacto que establezco entre mí y vosotros (...): pongo mi arco en las nubes para señal de mi pacto con la tierra (...). Estará el arco en las nubes y yo lo veré para acordarme de mi pacto eterno entre Dios y toda alma viviente y toda carne que hay en la tierra." (Génesis VIII, 12-16).

\section{Introducción}

Interesarse por la "Física" en abstracto, empieza por asombrarse antes por los fenómenos del mundo que nos rodea, y tratar de darles una explicación racional y coherente. El arco iris ha asombrado desde antiguo al ser humano hasta el extremo de llegar a considerarlo como la "firma de Dios", según se lee en el Génesis.

Si cuando enseñamos física, tratamos de despertar en nuestros estudiantes esta curiosidad y potenciarla hasta donde sea posible, no hay duda de que este hermoso fenómeno puede ser un punto de apoyo para profundas reflexiones, tantas que su completa explicación no se ha completado hasta hace pocos años, pero a ésta se puede llegar por pasos sucesivos. 
La mayoría de libros elementales de Física, y no tan elementales, u omiten el tema, que es lo más frecuente, o lo solucionan poniéndolo como ejemplo de la descomposición de la luz blanca por las gotas de agua, pero suelen quedarse ahí. En las líneas que siguen se trata de resumir lo que según muchos autores es todo un tratado de física, sacando el máximo partido didáctico posible a este hermoso fenómeno.

El trabajo se expone a un nivel medio a fin de darle su máxima utilidad a profesores de EE MM o de primeros años de carrera de ciencias y pretende recordar o, incluso, dar una primera explicación al docente no especialista, el autor tampoco lo es, y proporcionarle algunas pautas de trabajo en clase.

Lo ideal es partir de la observación directa del arco iris, pero esto no es siempre posible, entonces es útil disponer de fotografías o diapositivas.

Se recomienda utilizar película de bastante sensibilidad, unos 400 ASA, y un gran angular de $28 \mathrm{~mm}$. Para el cálculo, simulación y gráficos se ha utilizado la Hoja de Cálculo Excel, entre otras razones, debido a su amplia difusión, sencillez de uso y potencia operativa.

\section{Primeras observaciones}

A partir de la observación inicial surgen algunas preguntas como:

-¿Cuántos arcos hay?

-¿Qué colores y en qué orden aparecen en el arco iris?

-En caso de haber más de uno, ¿Cuál es su posición relativa: son concéntricos o se cruzan? ¿Aparecen los colores en el mismo orden? 
-Si aparecen dos arcos concéntricos, ¿Cómo es el color de cielo en la región intermedia?

-Cuando aparece un solo arco, ¿qué color tiene el cielo de fondo en sus proximidades?

-¿Cuál es la posición relativa entre el sol y el arco?

-¿Son siempre coloreados?

Responder con precisión a estas cuestiones no es sencillo y requiere siempre una observación minuciosa del fenómeno.

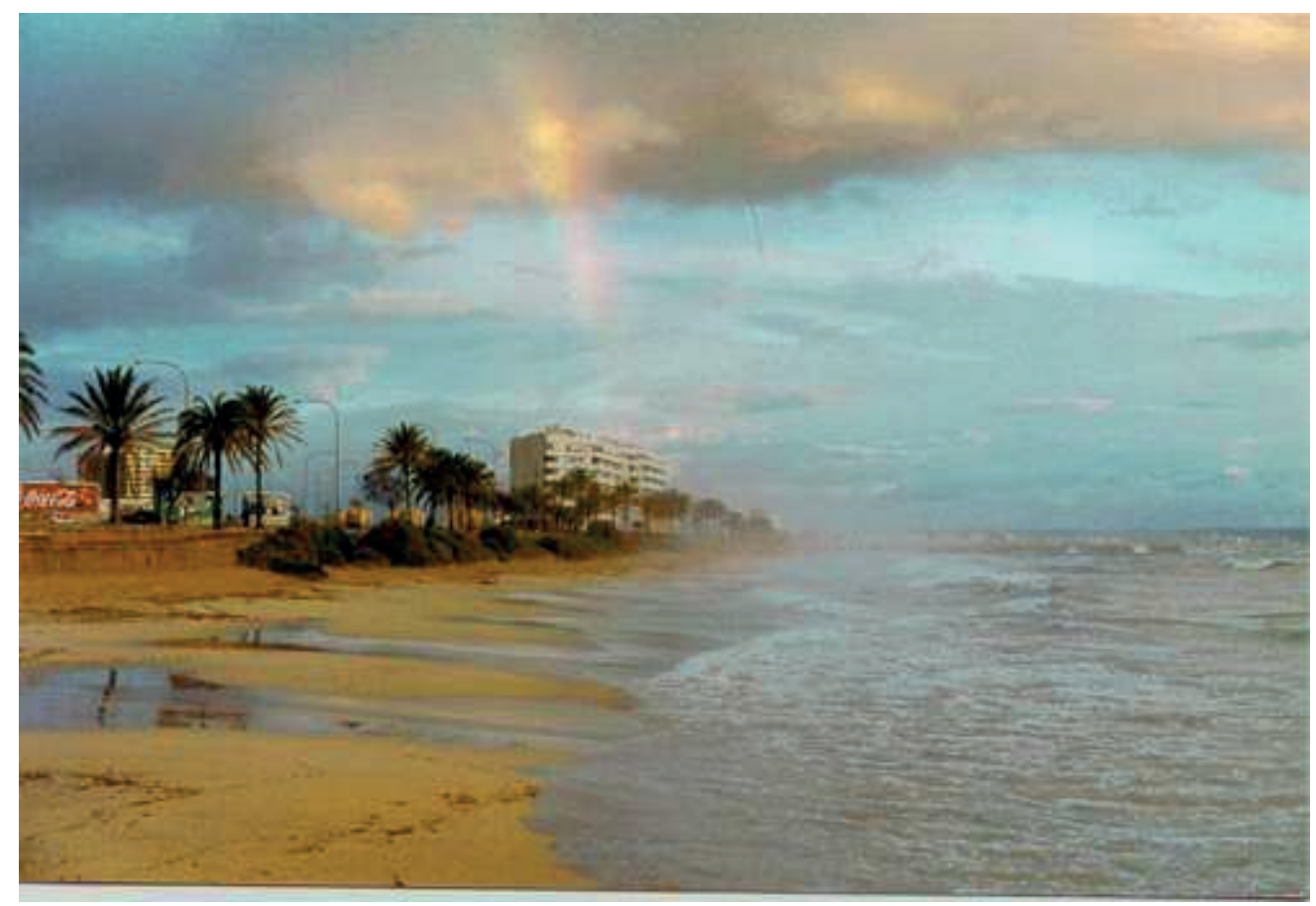

Lo normal es que no aparezca más que un solo arco, sin embargo, en algunos casos, se dan dos arcos el más "bajo" sobre el horizonte, algo más intenso que el más alto, con una zona intermedia más oscura. Al arco más intenso se le suele llamar primario, mientras que al otro se le llama secundario. La zona oscura es la llamada "zona de Alejandro", en honor a Alejandro de Afrodisias (s. II d. J.C.) filósofo griego que fue el 
primero en describirlo. Esta zona oscura aparece más difusa aunque no exista el arco secundario.

Las observaciones pueden hacerse algo más cuantitativas si se dispone de algún instrumento destinado a medir la altura de los objetos estelares. Los aparatos de precisión para estos menesteres son caros y difíciles de conseguir, sin embargo en nuestra práctica utilizamos una especie de teodolito casero, útil para múltiples observaciones, cuyo esquema se muestra en la figura 1.

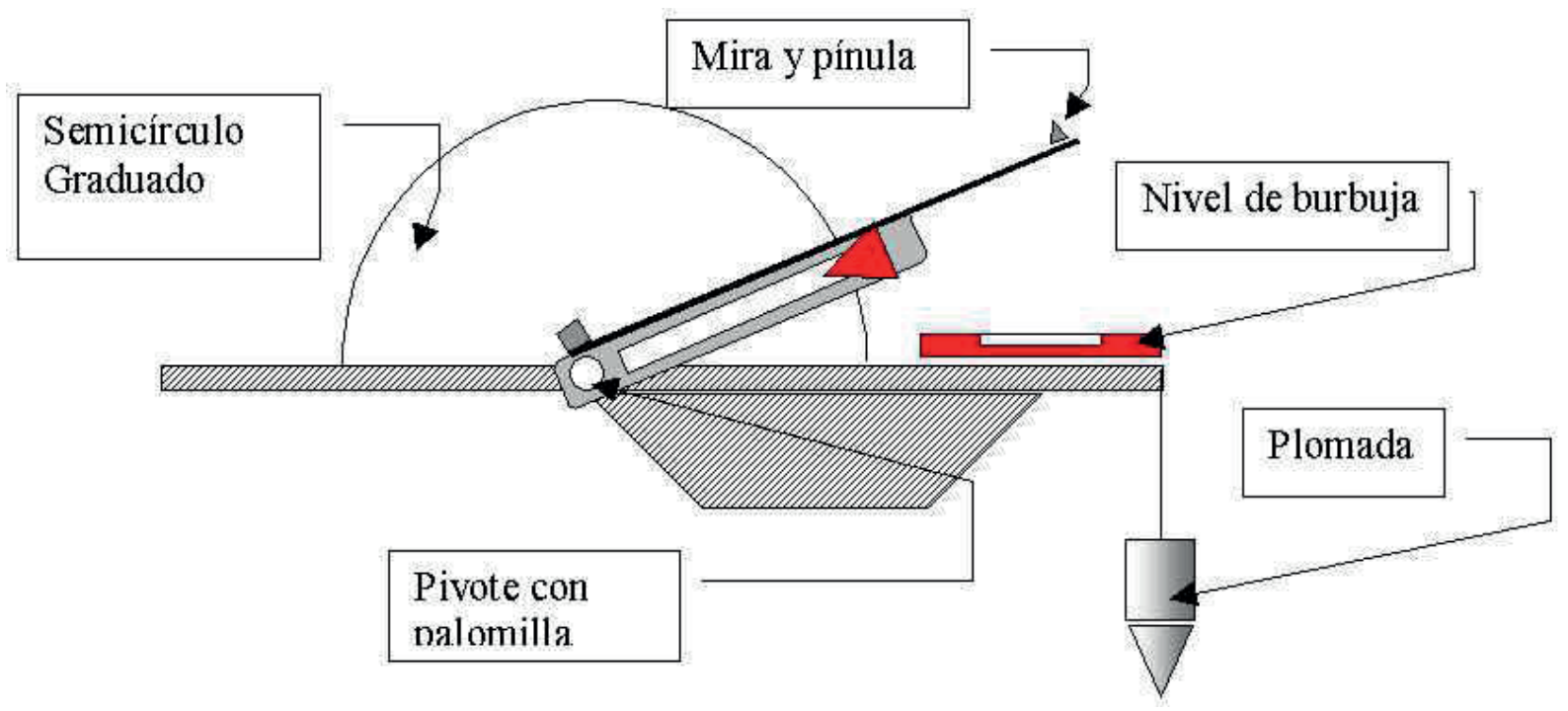

Figura 1

Consiste en un semicírculo graduado de plástico fijado con cianoacrilato a una tablilla o listón de aluminio. En el centro se sitúa la mira que es otro listoncillo de cualquier material, por ejemplo una regla de plástico, en el que se fijan mira y pínula. El conjunto pivota en torno a un tornillo que puede fijarse con una palomilla, para más sencillez en la medida. La plomada y el nivel tienen por objeto la correcta nivelación del aparato. 
Este "teodolito" puede fijarse en un trípode de fotografía o en un simple listón que se clava en el suelo.

Roger Bacon, 1266, midió por primera vez la altura del arco iris. El arco primario se encuentra a una altura de unos $42^{\circ}$ mientras que el secundario está a unos $50^{\circ}$, siempre con la horizontal como referencia. Entre ambos existe una separación de $\operatorname{unos} 8^{\circ}$.

Nosotros, en siete observaciones diferentes y para el arco principal, apuntando a la banda violeta con un instrumento como el descrito hemos obtenido en promedio $41,6^{\circ} \pm 1,6$

\begin{tabular}{|c|c|}
\hline Observación & Medida $/ \mathbf{(}^{\mathbf{}} \mathbf{)}$ \\
\hline 1 & 42 \\
\hline 2 & 44 \\
\hline 3 & 40 \\
\hline 4 & 42 \\
\hline 5 & 41 \\
\hline 6 & 43 \\
\hline 7 & 39 \\
\hline Media & 41,6 \\
\hline Desviación & 1,6 \\
\hline
\end{tabular}

Tabla 1. Altura del arco (Banda violeta)

El arco secundario, en condiciones de observación, sólo lo hemos visto 3 veces, obteniendo resultados de $51^{\circ}, 49^{\circ}$ y $48^{\circ}$

El arco primario presenta el rojo en el lado más alto, mientras que el violeta está en el más bajo, en el secundario ocurre lo contrario, el violeta está en la posición más alta y 
el rojo en la más baja, además en el lado violeta del arco primario aparecen algunos arcos violeta-azul, más o menos difusos, son los llamados arcos supernumerarios.

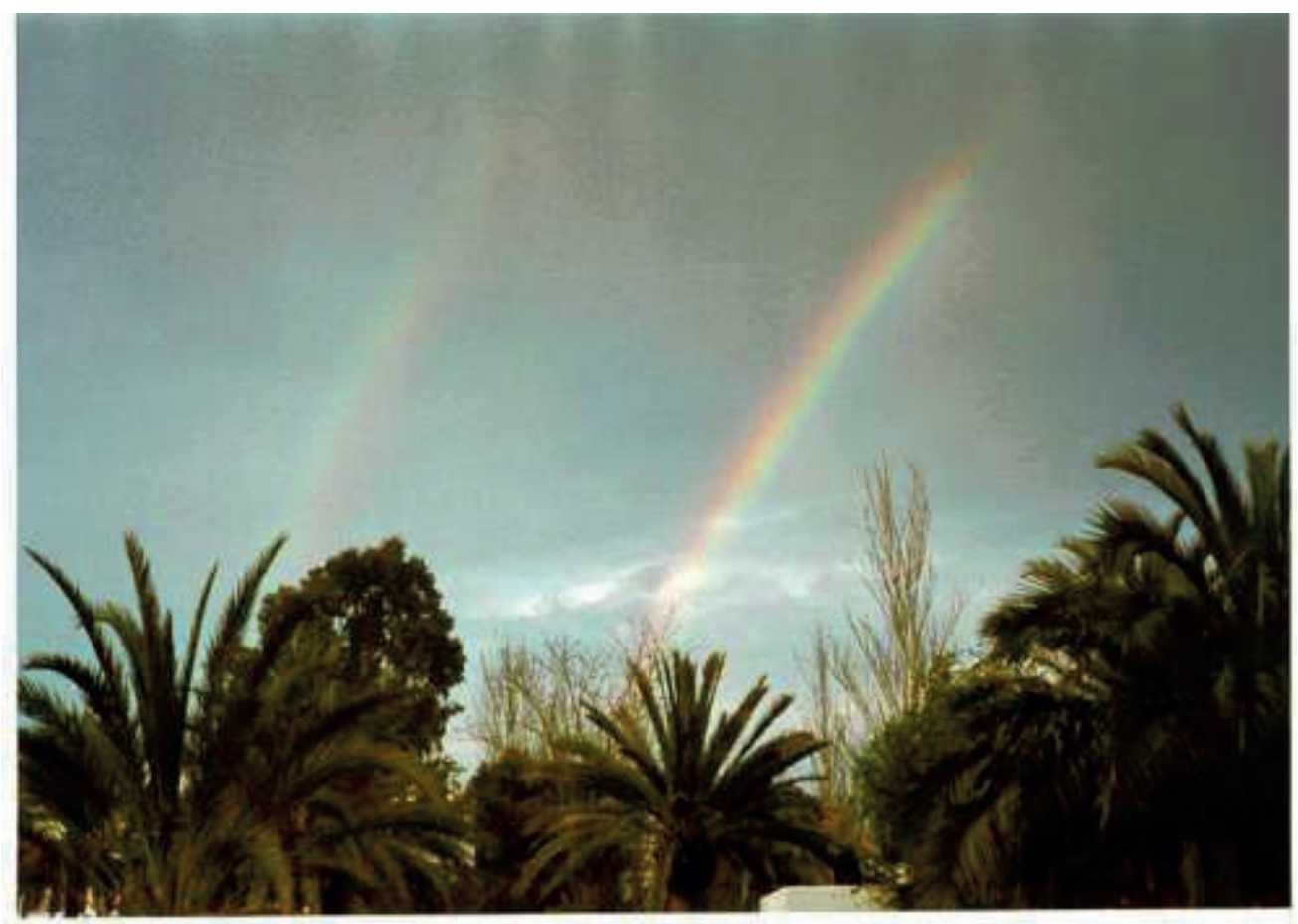

Arco iris doble desde distintos ángulos, obsérvese la inversión de los colores así como la zona de Alejandro.

Algunos autores han descrito "arcos blancos". Nosotros no los hemos observado nunca. Los arcos siempre se observan en dirección opuesta al Sol.

\section{Primer nivel de explicación}

Según Aristóteles, el arco iris se debería a una especial reflexión de la luz en las nubes, según un ángulo fijo, un cono circular de rayos de arco, que, entonces, no es un objeto propiamente dicho, sino una serie de direcciones privilegiadas de reflexión 
de la luz hacia el observador. En 1301 Teodorico de Freiberg, expuso que el arco iris podía formarse con una sola gota de agua y lo verificó con un frasco esférico lleno de agua, que venía a ser una gran gota. También el obispo de Spalato Antonio de Dominis, dio alguna explicación en esta línea. René Descartes, ignorando los trabajos de Teodorico, postuló la misma explicación, mostrando que el arco iris se produce gracias a los rayos que sufren una reflexión interna en la gota, mientras que arco secundario sufre dos reflexiones, siendo el ángulo de salida de los distintos colores diferentes, de modo que, si se observa en una sola dirección, sólo se ve un color, por lo que concluyó que los distintos colores procedían de gotas diferentes.

Un primer nivel de explicación es precisamente el propuesto por Descartes, que es posible actualizar, gracias a los medios de que hoy se dispone.

Cuando los rayos solares alcanzan la superficie de una gota de agua en parte se reflejan y en parte se refractan, es decir penetran en el interior de la gota donde a su vez sufren nuevas reflexiones y refracciones, esquemáticamente 


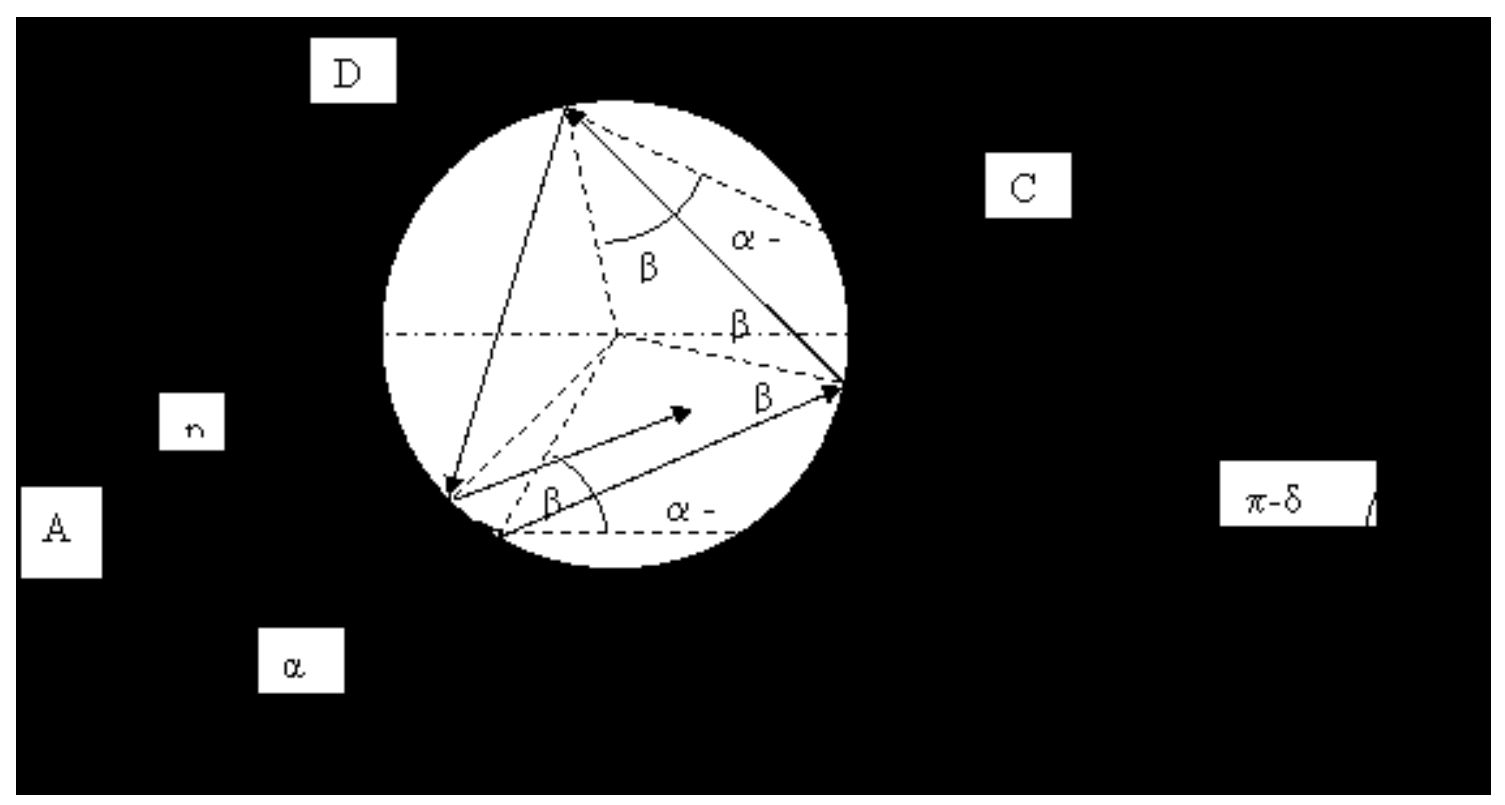

Figura 2

Un rayo incidente $\mathrm{A}$, cuya distancia al diámetro en la dirección del Sol recibe el nombre de parámetro de impacto, p, incide sobre al gota. Parte de él se refleja, rayo B y parte se refracta, siguiendo las leyes de Snell. Para al refracción sen $a=n$ sen $\AA$, siendo como es sabido a y $\mathbb{B}$ los respectivos ángulos de incidencia y refracción y $\mathrm{n}$ el índice de refracción del agua, aproximadamente 1,33. (El del aire se toma como aproximadamente igual a 1) El rayo refractado incide sobre la cara correspondiente de la gota, parte se refracta y emerge de la gota y parte se vuelve a reflejar. Simples consideraciones geométricas y la aplicación de las leyes de Snell a la reflexión permiten establecer la igualdad de los ángulos marcados y, en consecuencia, del ángulo con el que emerge el rayo $\mathrm{C}$, respecto el incidente. Como resultado el ángulo de desviación d vale

$d=180-(360-(2(a-\beta)-(360-2 ß)))=180-(4 \AA-2 a)$.

Descartes calculó las desviaciones de los rayos C en función del parámetro de impacto encontrando que presenta un mínimo para valor aproximado de $7 \mathrm{r} / 8$, 
siendo $\mathrm{r}$ el radio de la gota. Hoy es posible remedar los cálculos de Descartes en unos minutos utilizando una hoja de cálculo, en la que se programa un serie de valores del parámetro p y se obtienen los correspondientes valores de a y $ß$

En la figura 3 se representa la evolución de los valores de a, $\beta$ y d observándose el mínimo que corresponde a un valor del parámetro de 0,86 , con un ángulo de desviación de $138,5^{\circ}$ es decir, totalmente coincidente con el hallado por Descartes.

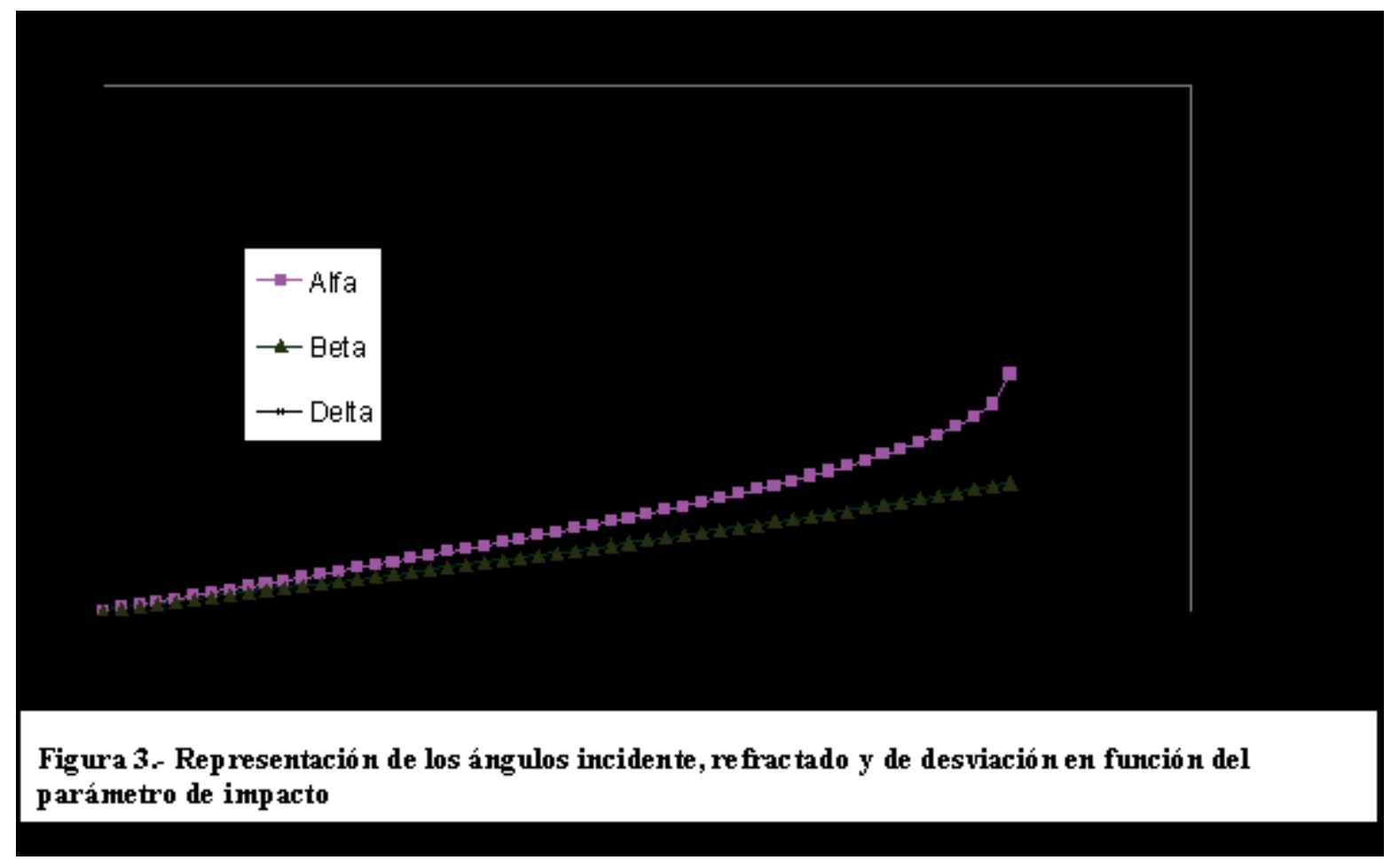

Jugando una vez más con la hoja de cálculo, se puede observar si el índice de refracción varía ligeramente, como realmente ocurre con la "luz blanca"NOTA1, la desviación de los rayos para el mismo parámetro es ligeramente diferente, figura 4, en la que se representa el ángulo de desviación para valores de índice de refracción 1,30; 1,32; 1,34 respectivamente frente al parámetro de impacto. 
En cuanto al rayo procedente de la segunda reflexión, E, su desviación se puede calcular mediante sencillas consideraciones geométricas, $\mathrm{d}^{\prime}=6 \mathrm{~b}-2 \mathrm{a}$. Una vez más, recurriendo a la Hoja de Cálculo se observa que su comportamiento es inverso a d, es decir, crece y presenta un máximo también aproximadamente a 0,96r y con un

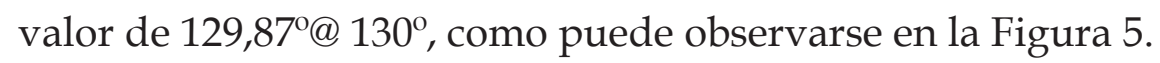

Se producen más reflexiones internas, que podrían dar lugar a nuevos arcos, sin embargo, en cada paso, se pierde energía, de modo que las reflexiones de orden superior al segundo dan origen a rayos emergentes muy débiles, por tanto a arcos tan tenues, que resultan invisibles (Ver NOTA 1).

En resumen, los resultados de ambas simulaciones confirman el resultado experimental dado que la altura del respectivo arco, suplementario del ángulo de desviación es el esperado. No obstante, todavía quedan puntos por esclarecer, por ejemplo, sólo se han tratado los rayos incidentes solares directos pero a la gota llegan, además de éstos, multitud de rayos dispersos procedentes de reflexiones con otras gotas o, en general, como consecuencia del esparcimiento (NOTA 2) de la luz por la atmósfera.

Los rayos que han sufrido las reflexiones internas se desvían en un campo muy amplio de direcciones, ¿por qué precisamente aquellos situados en las vecindades de la máxima o mínima desviación intensifican su intensidad? 


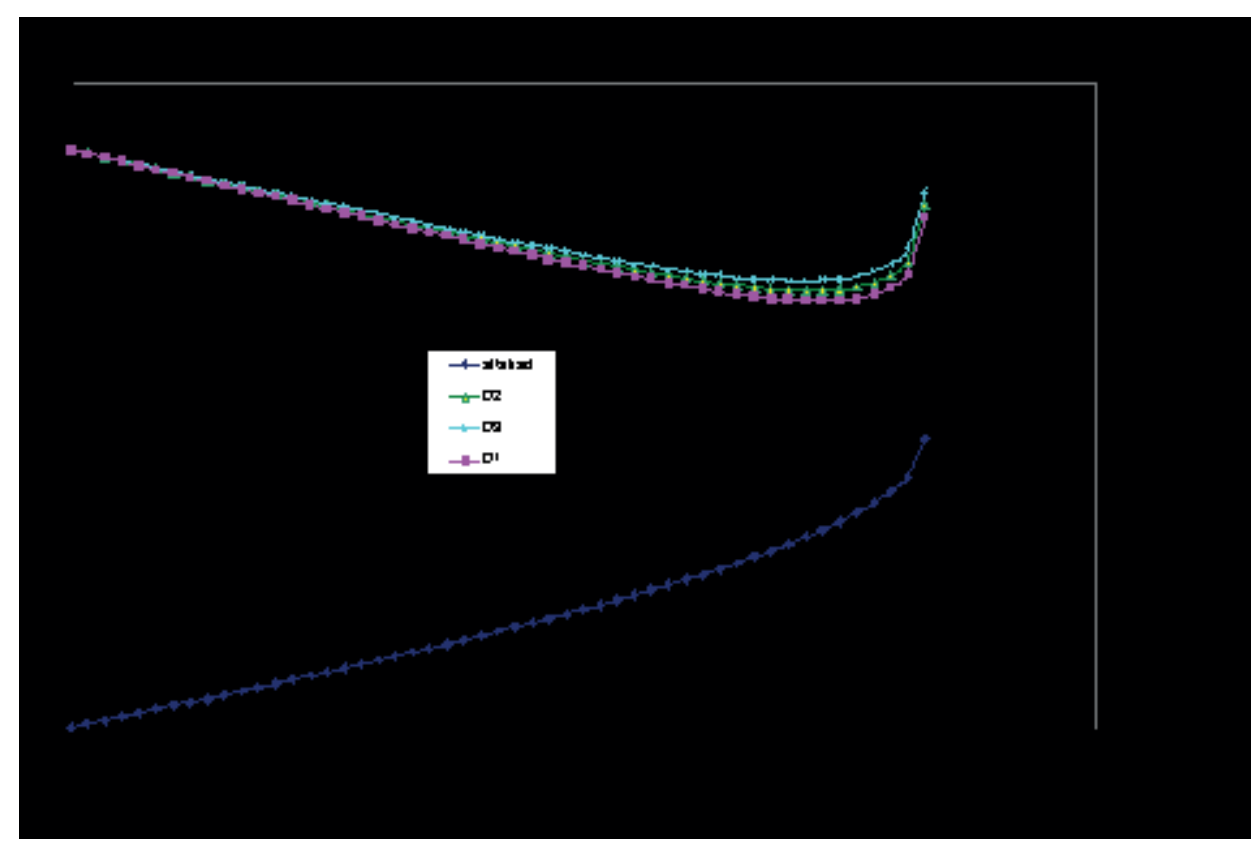

Figura 4: Ángulos de desviación e incidencia para distintos valores del índice de refracción

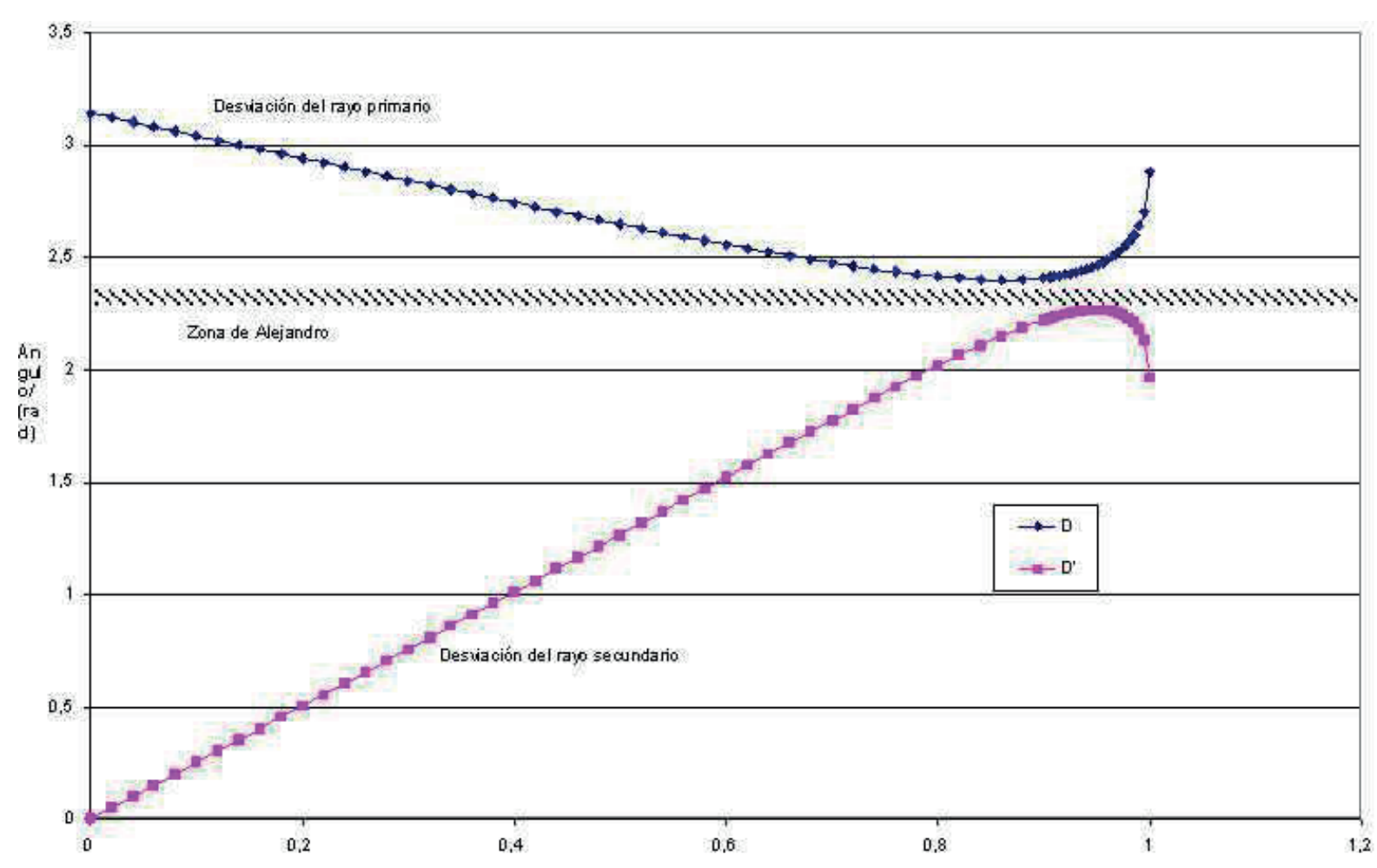


Figura 1: Ángulos de desviación de los rayos primario y secundario en función del parámetro de impacto.

La primera cuestión puede resolverse pensando que los rayos dispersos son de muy baja intensidad, comparados con la luz directa y, en consecuencia, los arcos que producirán serán muy tenues.

Respecto a la segunda cuestión, se puede responder que el primer rayo reflejado B, no ha sufrido descomposición, en consecuencia no formará arcos, el rayo C es el rayo refractado por la gota y se aleja del observador, la intensidad los rayos $\mathrm{C}$ y E, se refuerza en las vecindades del rayo cartesiano, ya que la gota está uniformemente iluminada en su cara enfrentada al Sol, el ángulo de desviación en principio disminuye (o aumenta) al aumentar el parámetro de impacto, sin embargo, al aproximarse al valor extremo, este aumento o disminución es cada vez más lento, de modo que en las proximidades de este valor se "acumulan " muchos más rayos que para los restantes valores, además superado el valor del extremo los rayos se desvía hacia atrás de la gota. En resumidas cuentas, pues, en las direcciones de $130^{\circ}$ y $138^{\circ}$ hay una acumulación de rayos. Además en la zona comprendida entre los $130^{\circ}$ y 138 ${ }^{\circ}$ (siempre en términos aproximados), no llegará ningún rayo después de la primera o segunda reflexiones, (Figuras 4 y 5 )

De este modo se explican, pues, la formación del rayo y la forma del arco.

Debido a la simetría de la gota, no existe un plano privilegiado, el observador recibirá los dos conos de luz, y la zona oscura que corresponderá precisamente a esta región de desviación entre $138^{\circ}$ y $130^{\circ}$. 
El tamaño de la gota no interviene en la formación de la arco, es posible reproducir la formación del arco, como ya lo hiciera en su momento Descartes, con una gran gota consistente en un balón de vidrio lleno de agua. NOTA 3.

Queda por explicar la parte más espectacular del rayo, sus colores. Debido a la diferencia de los índices de refracción en el agua para los distintos colores, éstos se desvían de modo diferente. El índice de refracción, que como es sabido es la relación entre la velocidad de la luz en el medio y en el vacío (@ aire), en el agua es menor cuando menor es la longitud de onda, es decir, cuando más próxima al rojo es la luz, más lentamente se propaga en el agua, y una vez más la simulación en la hoja de cálculo, sólo con tres "colores" por obvias razones de claridad y sencillez, pone de manifiesto que la desviación es mayor o lo que es lo mismo, la altura sobre el horizonte, será menor cuando más próxima al violeta se halle la radiación, viceversa con el secundario.

Newton había calculado que el ángulo de desviación era de $137^{\circ} 58^{\prime}$ para el rojo y $139^{\circ} 43^{\prime}$ para el violeta, lo que da para el arco primario una amplitud de $1^{\circ} 45^{\prime}$, pero, dado que el Sol no es un punto sino que tiene una amplitud de aproximadamente medio grado, la amplitud del arco primario será de unos $2^{\circ} 15^{\prime}$. Con el "teodolito casero" es muy difícil estimar esta amplitud, pero trabajando con cuidado se obtienen amplitudes de entre $2^{\circ}$ y $3^{\circ}$. 


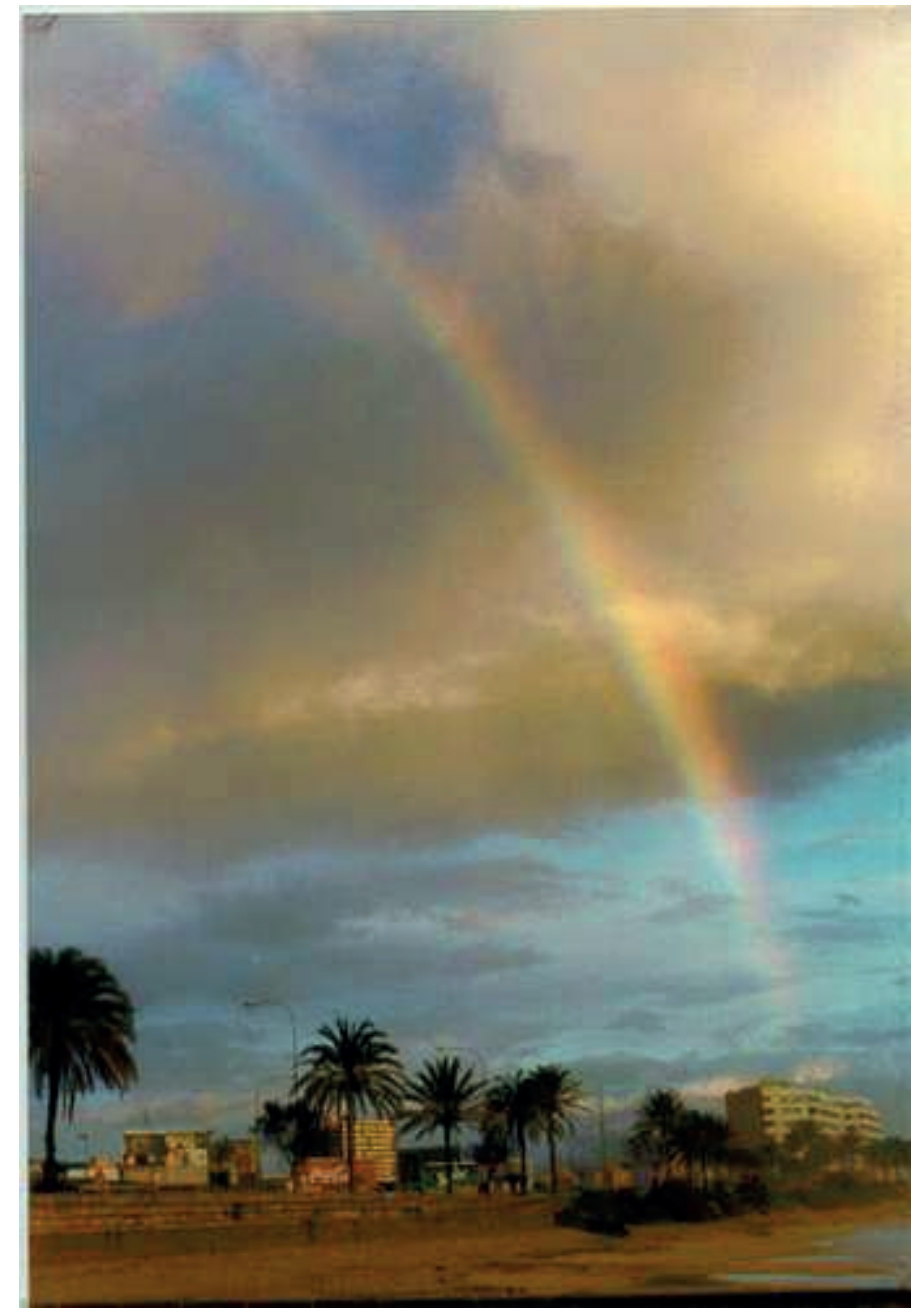

Dada la simetría de la gota, aparecerán dos rayos cartesianos, uno a cada lado, de los que uno enviaría el rayo formador del arco "hacia arriba". Este arco no podría ser visto por el observador en el suelo, pero ¿lo sería por alguien que volara por encima de la lluvia? En caso afirmativo ¿Qué forma tendría? Todo parece indicar que debería ser un "arco al revés". Hemos interrogado a amigos pilotos pero sólo han visto arcos "con los cuernos hacia abajo", sin recordar a qué altura volaban. Ahí queda la cuestión con la esperanza de que algún aviador aficionado a la física nos dé alguna respuesta.

Esta explicación, que no requiere conocimientos muy elevados hace posible explicar de modo riguroso los aspectos fundamentales del arco iris, incluso con un cierto 
grado de cuantitatividad, pero quedan algunos aspectos más difíciles, como los arcos supernumerarios y los "arcos blancos" o el que el arco no se corte de modo brusco en la banda oscura. Según esta teoría ningún rayo, al menos de clase B o C, debería llegar a la zona de Alejandro, que debería, además y en consecuencia, ser muy oscura, esto no es así.

\section{Segundo nivel de explicación}

Una primera explicación a los arcos supernumerarios se debe a Young (1803), que se basa en su propia teoría de la interferencia. Si se observa la figura 4 , se ve como en las vecindades del rayo cartesiano, se encuentran siempre dos rayos uno a cada lado del cartesiano, que, pese a haber seguido caminos ópticos diferentes dentro de la gota, emergen con el mismo ángulo de desviación. Con los conocimientos actuales de mecánica ondulatoria se sabe que las intensidades de estos arcos no pueden sumarse algebraicamente, sino que es un fenómeno idéntico al conocido experimento de las dos ranuras del propio Young Como es sabido cuando coinciden dos ondas se interfieren, esta interferencia puede reforzar la intensidad, interferencia constructiva, el máximo se da cuando la diferencia de caminos es un número par de semilongitudes de onda o debilitarla, incluso anularla, cuando la diferencia de caminos es un número impar de longitudes, destructiva. Los alumnos pueden con facilidad simular las interferencias con la hoja de cálculo. (Fig. 6). 


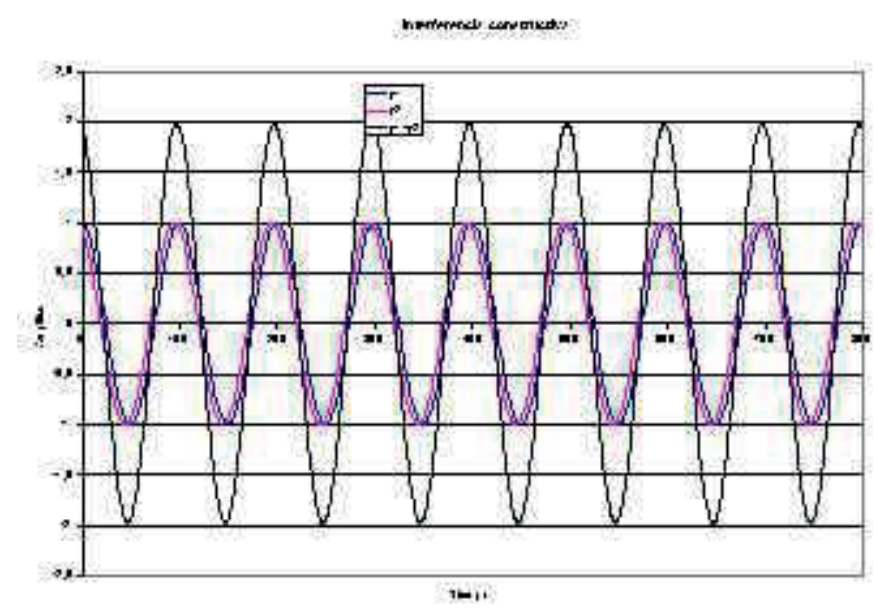

Figura 6. Interferencia constructiva.

Así, cuando la diferencia de los ángulos es pequeña, los caminos ópticos en el interior de la gota son muy parecidos y la interferencia es constructiva.

El resultado de estas interferencias constructivas son precisamente los arcos supernumerarios. Evidentemente, aquí si es importante el radio de la gota porque el camino óptico depende de él. Un poco de geometría pone de manifiesto que este camino es

$e=4 r \cos \beta=\frac{4 r}{n} \sqrt{n^{2}-p^{2}}$

siendo $\mathrm{r}$ el radio de la gota, $\mathrm{n}$ el índice de refracción del agua para la radiación implicada y p el parámetro de impacto, como fracción del radio de la gota.

Por otra parte, para una misma gota la separación entre pares de rayos homólogos a su vez va creciendo de modo que inicialmente la interferencia es constructiva, con una intensidad muy grande, es el arco iris, pero la intensidad de la resultante va disminuyendo, hasta que se hace absolutamente destructiva, lo que correspondería a 
una banda oscura a partir de ahí vuelve a aumentar la intensidad de la interferencia, hasta otro absolutamente constructiva, aun bastante intenso, este primer máximo corresponde al primer arco suplementario y así sucesivamente.

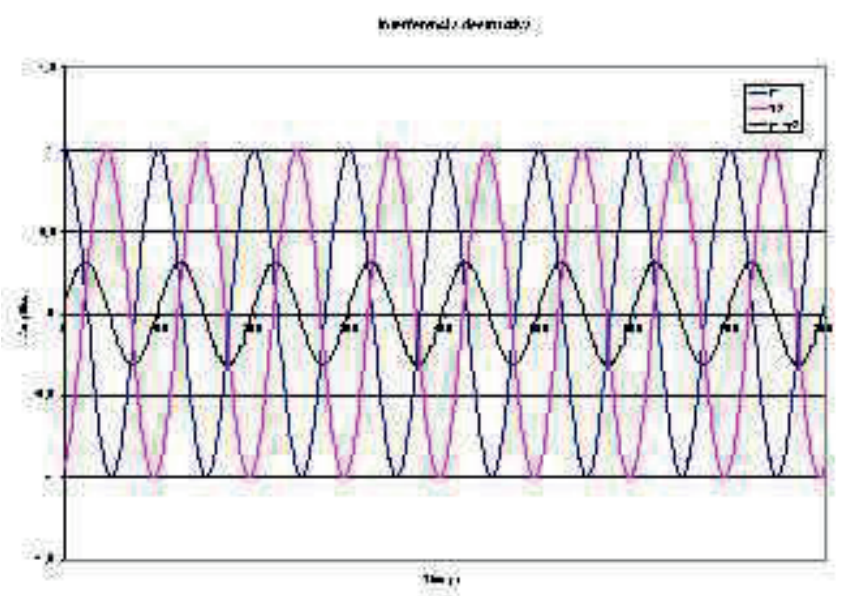

Figura 7. Interferencia destructiva.

Por otra parte, es fácil comprobar, y una vez más la Hoja de Cálculo es una excelente ayuda, que para pares de parámetros homólogos, la separación entre los caminos crece con el radio de la gota, en consecuencia las secuencias de máximos y mínimos pueden quedar muy próximas y los arcos no se distinguirán. (La frontera está aproximadamente en $1 \mathrm{~mm}$ ). En efecto, los arcos suplementarios se verán mejor en la parte alta del arco, donde las gotas son menores, que en la baja donde son más grandes. Respecto al índice de refracción, la separación aumenta más rápidamente para valores de índice de refracción menores, es decir, para aquellas radiaciones que tienden al violeta, de ahí la coloración rosada de los arcos supernumerarios. Cuando la separación entre los arcos supernumerarios sea muy pequeña, pueden "mezclarse" dando un color blanco, de hecho, en general, son rosados, no rojos, o verdosos.

La teoría de Young explica la formación de arcos supernumerarios pero, en cierto sentido, implica una contradicción en si misma ya que, si las ondas se interfieren, 
también se difractan y, como consecuencia, la separación entre las distintas zonas del arco debe ser suavizada por la difracción.

Potter (1935) propuso una idea según la cual el cruce de varios conjuntos de rayos luminosos en una gota daba lugar a una curva cáustica, este tipo de curvas son las envolventes de un conjunto de rayos, una típica es la que se observa cuando el sol se refleja en una taza y da lugar a dos curvas brillantes que se unen en punta, con una intensidad creciente hasta la curva para entrar bruscamente en una zona oscura. En cualquier caso tanto la explicación de Descartes como las de Young y Potter predicen en el rayo cartesiano una intensidad infinita.

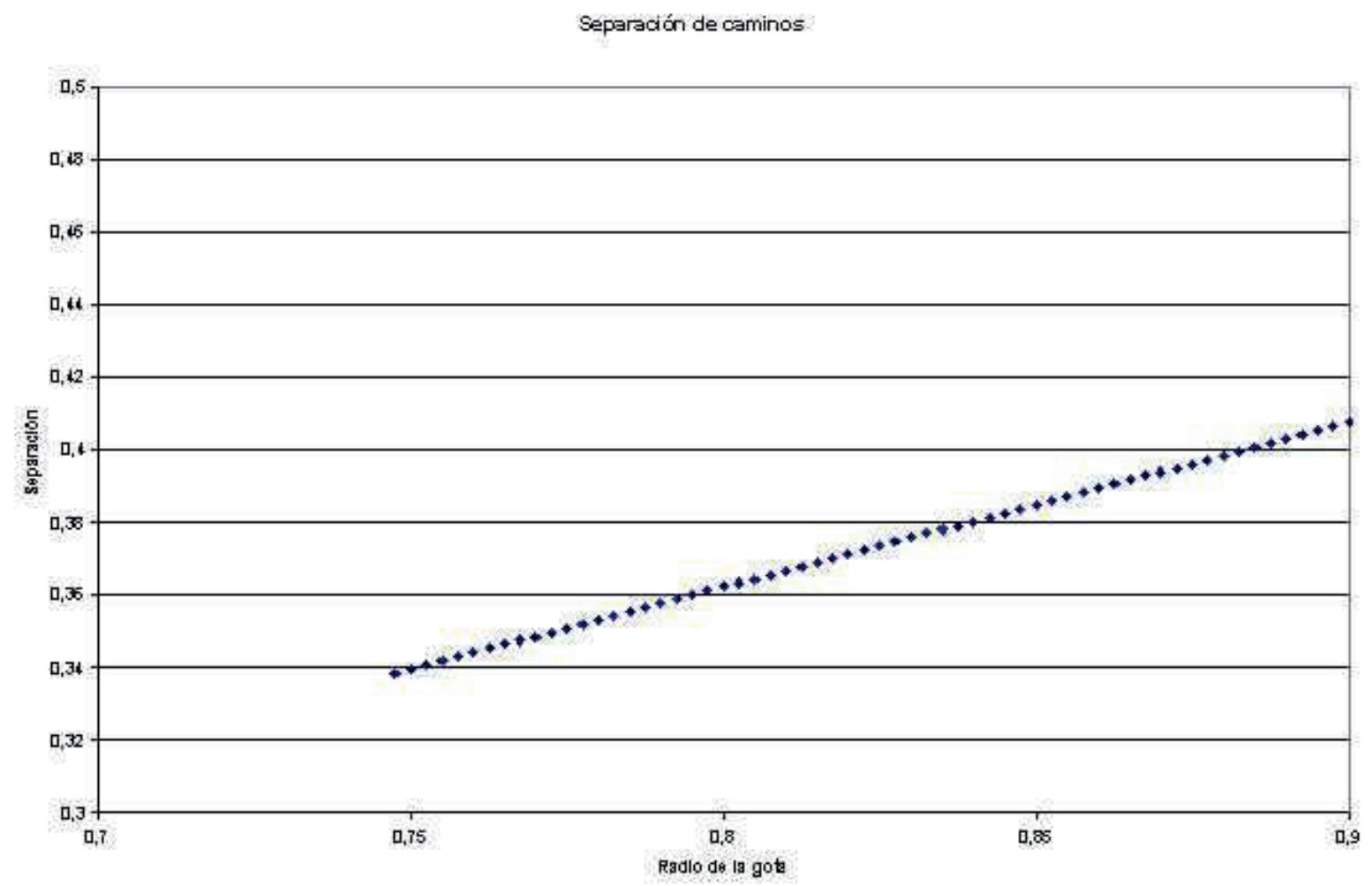

Figura 8: Ritmo de crecimiento de la separación entre los caminos en función del radio de la gota.

\section{Tercer nivel de explicación}


Esta explicación se debe a Airy, allá por 1838, quien partiendo de los conocimientos que ya se tenían en su época de las propiedades de las ondas, especialmente del principio de Huyggens, según el cual, todo punto alcanzado por una onda se convierte a su vez en foco emisor de ondas esféricas, siendo la envolvente de estas ondas el nuevo frente resultante. Si en un instante dado se conoce un frente de ondas y las amplitudes de las ondas secundarias a él asociadas, es posible predecir el comportamiento de la onda en cualquier punto. Sin embargo, este análisis no es rigurosamente posible, sólo cabe estimar estas amplitudes. Airy propuso un frente de ondas en el interior de la gota, normal a todos los rayos clase $\mathrm{C}$ y con un punto de inflexión en su corte con el rayo cartesiano. Así pudo predecir la intensidad de la luz del arco iris por medio de una función, que hoy se conoce como función de Airy y que él llamó integral del arco iris. Está función bastante compleja, aunque deducida de los conocimientos generales y clásicos de la teoría de las ondas, predice un máximo relativo correspondiente al arco primario y máximos menos fuertes para los arcos supernumerarios, pero también existe en la zona oscura, de modo que explica su atenuación gradual. Los máximos se desplazan ligeramente respecto a la teoría de Young y en ningún momento se predice una intensidad infinita.

La teoría de Airy se aplica en rigor a un arco monocromático y puede verificarse experimentalmente con un láser de bolsillo y un gota de agua en la punta de una jeringa o una bureta. Los rayos policromos deberían considerarse como yuxtaposición de varios monocromáticos, ahí el tamaño de la gota es decisivo, gotas de unos cuantos milímetros producen arcos claros y brillantes, en gotas muy pequeñas $(\varnothing<0,01 \mathrm{~mm})$ los arcos aparecen tan juntos que dan la sensación de "blancos", no debe olvidarse que la percepción del color traspasa la mera física para entrar en la fisiología, y que el sentido de la vista, al contrario del oído, es sintético.

\section{Conclusión}


Hasta ahora se ha visto como las explicaciones se van aproximando sucesivamente a una explicación definitiva. Esta desde luego existe, incluso llegando hasta el nivel molecular, lo que significaría "arcos iris atómicos", pero incluso a nivel macroscópico, sin entrar en conceptos mecánico cuánticos, y utilizando las ecuaciones de Maxwell, es posible una explicación completa del fenómeno, ello no obstante, dado el nivel a que va dirigido este trabajo, parece fuera de lugar puesto que requeriría el manejo de conceptos físicos con los cuales estudiantes de bachillerato o primer año de carrera no están familiarizados.

\section{Agradecimientos}

A mis colegas la Dra. Carmen García Domingo del C.P.R. de Burgos y a Francisco Alejandro Vinagre Benito, por sus inapreciables consejos y al Cmte. Piloto D. Antonio Morillo, por sus atinadas observaciones.

\section{Notas}

NOTA 1: En el laboratorio, con un poco de buena mano, es posible conseguir varios arcos. Véase el artículo de Jearl Walker, en Investigación y Ciencia, Septiembre de 1977: "Como crear y observar una docena de arcos iris en una sola gota de agua".

NOTA 2: "scattering"

NOTA 3: Por honestidad científica hay que decir que conseguir unos resultados aceptables no es siempre fácil y que, en nuestro modesto entender, debe descartarse el experimento como "práctica de cátedra". 